\title{
Surface Energy Transfer in Hybrid Halide Perovskite/Plasmonic Au Nanoparticle Composites
}

Susana Ramos-Terrón,,$+{ }^{\star}$ David Alba-Molina,,,,$+{ }^{\star}$ Maria de los Ángeles VaroSantos, ${ }^{\dagger}$ Manuel Cano, ${ }^{\dagger}$ Juan José Giner-Casares ${ }^{\dagger}$ and Gustavo de Miguel ${ }^{\dagger}$

† Departamento de Química Física y Termodinámica Aplicada, Instituto Universitario de Investigación en Química Fina y Nanoquímica, IUNAN, Universidad de Córdoba, Campus de Rabanales, Edificio Marie Curie, E-14071 Córdoba, Spain

$\ddagger$ Currently at the Maimónides Institute of Biomedical Research (IMIBIC), Reina Sofía University Hospital, Córdoba, Spain

* These authors contributed equally to this work

\section{Corresponding Author}

E-mail: jiginer@uco.es and gmiguel@uco.es 


\section{Abstract}

Incorporation of plasmonic metal nanoparticles (NPs) into the multilayered architecture of perovskite solar cells (PSCs) has been a recurrent strategy to enhance the performance of the photovoltaic devices from the early development of this technology. However, the specific photophysical interactions between the metal NPs and the hybrid halide perovskites are still not completely understood. Herein, we investigate the influence of Au NPs on the photoluminescence (PL) signal of a thin layer of the $\mathrm{CH}_{3} \mathrm{NH}_{3} \mathrm{Pbl}_{3}$ hybrid perovskite. Core-shell Au@SiO 2 NPs with tunable thickness of the $\mathrm{SiO}_{2}$ shell were used to adjust the interaction distance between the plasmonic NPs and the perovskite layer. A complete quenching of the PL signal in the presence of the Au NPs is measured together with a gradual recovery of the PL intensity at thicker thickness of the $\mathrm{SiO}_{2}$ shell. A nanometal surface energy transfer (NSET) model is employed to reasonably fit the experimental quenching efficiency. Thus, the energy transfer deactivation is revealed as a detrimental process occurring in the PSCs since it funnels the photon energy into the non-active excited state of the Au NPs. This work indicates that tuning the distance between the plasmonic NPs and the perovskite materials by a silica shell may be a simple and straightforward strategy for further improving the efficiency of the PSCs. 


\section{Introduction}

The advent of the hybrid organic-inorganic halide perovskites as absorber material in solar devices has caused an incredible impact on the field of material science in the last few years. ${ }^{12}$ The extraordinary optoelectronic properties and ease of preparation of the halide perovskites has encouraged their implementation in several applications as, for example, light-emitting devices, ${ }^{3,4}$ photodetectors, 5,6 lasing ${ }^{7,8}$ and photocatalysis., 90 Additionally, the hybrid perovskites have been combined with other advanced materials to enhance their optoelectronic features or to develop innovative applications. Thus, twodimensional hybrid perovskites have been merged with chiral organic molecules to develop spintronics and quantum computing ${ }^{11}$ or with graphene aiming at improving the stability and performance of photodetectors and photocatalysts. ${ }^{12}$ In particular, one of the first approaches to enhance the performance of perovskite solar cells (PeSCs) was to incorporate core-shell gold-SiO 2 nanoparticles into the perovskite layer. ${ }^{13}$

Plasmonic nanoparticles (NPs) are characterized by the resonance between the oscillation of the surface electrons and the coherent electromagnetic radiation, namely the so-called plasmonic effect. ${ }^{14}$ Plasmonic NPs exhibit strong UV-visible optical absorption and far-field light scattering which are strongly dependent on the size and shape of the NPs. ${ }^{15}$ Thus, the incorporation of plasmonic metal NPs in PeSCs may improve the light harvesting in these devices and therefore, facilitate the reduction of the amount of toxic $\mathrm{Pb}$ atoms in the solar cells. ${ }^{16,17}$ However, the published articles typically report minor improvements of the device performance when core-shell Au or Ag NPs are incorporated into the active layer or hole transporting layers of PeSCs. ${ }^{18-20}$ Moreover, although enhancement of light absorption is the dominant explanation employed in organic solar cells (OSCs) for the increased performance, other different mechanisms have been proposed in PeSCs to account for the improved power conversion efficiency (PCE) associated to a higher short-circuit photocurrent $\left(\mathrm{J}_{\mathrm{sc}}\right) .{ }^{21-24} \mathrm{~A}$ reduction of the exciton binding energy $\left(E_{\mathrm{b}}\right)$ with a concomitant increase in the generation of free charge carriers has been suggested to explain the performance increase in PeSCs with Au@SiO2 NPs added to the photoactive layer. ${ }^{13,25}$ A novel photon 
recycling scheme consisting in a faster radiative decay of the excitons together with a reabsorption of the emitted light has been proposed to account for an

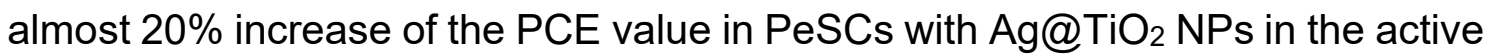
layer. ${ }^{19}$

Despite the reported improvement of the performance of PeSCs by using plasmonic NPs, there are certain evidence pointing out that plasmonic NPs might also interact with the hybrid perovskites via charge recombination or exciton quenching. ${ }^{23}$ In particular, it is described in the literature that the incorporation of plasmonic NPs may yield a decrease of the photoluminescence (PL) which is not easily explained by the light harvesting mechanism. ${ }^{13,18,26}$ Different tentative mechanisms have been proposed to account for the exciton quenching even though they are not well understood and require additional experimental investigations.

In this article, we explore the dependence of the PL intensity of a thin film of the archetypal $\mathrm{CH}_{3} \mathrm{NH}_{3} \mathrm{Pbl}_{3}\left(\mathrm{MAPb}_{3}\right.$ ) hybrid perovskite on the spacing to Au NPs using different thicknesses of $\mathrm{SiO}_{2}$ in the core-shell $\mathrm{Au} @ \mathrm{SiO}_{2} \mathrm{NPs}$. The interactions between the perovskite layer and the Au NP layer were non-specific, i.e., the perovskite layer was built on top of the Au NP layer. There were no specific or directed chemical interactions between the silica surface and the growing perovskite layer. The film thickness of the mixed layer was determined by the experimental conditions with no influence of the thickness of the silica layer coating the individual Au NPs. Note the value of thickness of the silica layer was tuned to control the distance between the surface of the Au NP and the perovskite material. The steady-state absorption spectrum of a thin film of plasmonic Au NPs displays a red tail overlapping with the $\mathrm{PL}$ spectrum of the $\mathrm{MAPbl}_{3}$ perovskite. This fact suggests the activation of an energy transfer process that might explain the PL quenching observed in many PeSCs. Thus, the quenching efficiency measured for different thicknesses of the $\mathrm{SiO}_{2}$ shell in the Au NPs is simulated with a nanometal surface energy transfer (NSET) model obtaining good agreement with the experimental results. 
The reported studies on hybrid perovskites/metal NPs composites display a PL quenching dependent on the metal employed in the NPs, nature of the shell and position of the metal NPs in the multilayered structure of the PeSCs. ${ }^{19,26,27}$ In this study, we have deposited both materials as homogeneous thin layers on top of each other to fine control the distance between the Au NPs and the MAPbl 3 perovskite. Scheme 1 displays the arrangement of the two layers with the plasmonic metal NPs deposited on top of a glass substrate through a spray pyrolysis method and a $100 \mathrm{~nm}$ thickness layer of the $\mathrm{MAPbl}_{3}$ perovskite prepared with the spin-coating method lying on the NPs film. The low thickness of the MAPbl 3 layer and the penetration of the MAPbl 3 in the NP layer guarantees that the majority of the photoinduced excitons or electron-hole carriers generated in the perovskite may diffuse to interact with the metal NPs before charge recombination since the carrier diffusion lengths are larger $\left(L_{D} \approx 100 \mathrm{~nm}\right){ }^{28}$

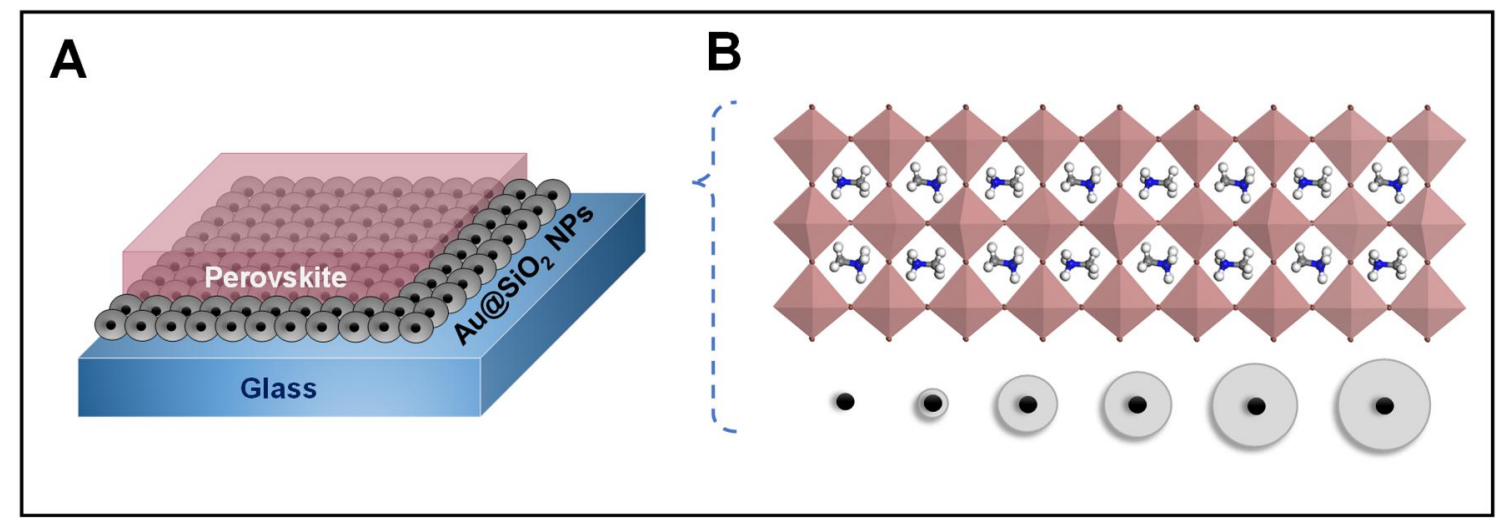

Scheme 1. A) Layout of the Au@SiO 2 NPs and $\mathrm{MAPb}_{3}$ hybrid perovskite layers. B) Detailed crystalline structure of the $\mathrm{MAPbl}_{3}$ hybrid halide perovskites with the methylammonium (MA) cation inserted into the octahedral voids and the Au NPs with different thicknesses of the $\mathrm{SiO}_{2}$ shell.

Figure 1 exhibits the UV-vis absorption spectra of the Au NPs in DMF solution and deposited as a thin layer before adding the MAPbl 3 perovskite film. The UVvis absorption spectra in solution and in thin layers display the characteristic plasmon peak centered at $525 \mathrm{~nm}$ related to the surface plasmon resonance (SPR) of a $20 \mathrm{~nm}$ diameter Au NPs. We do not observe any additional significant absorption signal in the thin layers due to potential plasmon coupling of the 
adjacent NPs, indicating reduced or no aggregation of the NPs. ${ }^{29,30}$ Figure 1 also displays the PL spectrum of a film of the MAPbl 3 hybrid perovskite with the typical PL peak centered at $765 \mathrm{~nm}$ (see Figure S4 for the XRD pattern). The low energy region of the absorption spectrum of the Au NPs (700-800 nm) overlaps with the $\mathrm{PL}$ signal of the MAPbl 3 perovskite which might enable a surface energy transfer mechanism as the process responsible for a non-radiative recombination of the excited charge carriers. A high quenching efficiency has been previously reported for CdTe quantum dots (QDs) with different sizes assembled in close proximity with Au NPs. ${ }^{31}$ The shift of the PL of the CdTe QDs with different sizes modifies the overlapping with the absorption of the Au NPs but the quenching efficiency was very high in all cases. ${ }^{31}$

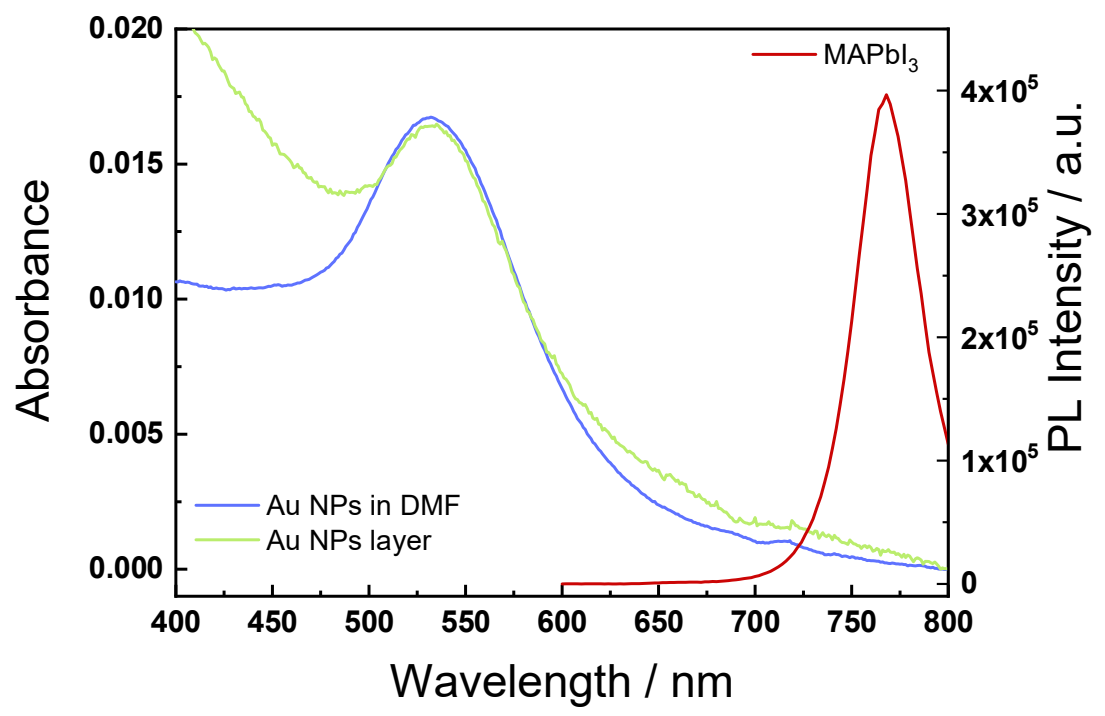

Figure 1. Absorption spectra of Au NPs in DMF solution (dark-blue line), Au NPs deposited as a thin layer (green line) and PL spectrum of the $\mathrm{MAPbl}_{3}$ hybrid perovskite film (red line). $\lambda_{\text {exc }}=400 \mathrm{~nm}$.

Core-shell Au@SiO 2 NPs with tunable thickness of the optically inert $\mathrm{SiO}_{2}$ shell were synthesized by using the Stöber method to examine the dynamics of the photophysical deactivation process in the $\mathrm{MAPbl}_{3}$ perovskite at controlled distance to the Au NPs (see further details of the synthesis in the supporting information, Figure S1-S3). ${ }^{32-34}$ Figure 2 shows representative TEM images of the core-shell $\mathrm{Au} @ \mathrm{SiO}_{2} \mathrm{NPs}$ where a clear rise in the $\mathrm{SiO}_{2}$ thickness is observed upon increasing the duration of the Stöber growth. Figure S5 exhibits the UV-Vis absorption spectra of all core-shell Au@ $\mathrm{SiO}_{2} \mathrm{NPs}$ in bulk DMF solution where a 
small red-shift of the maximum of the absorption peak is detected at increasing thickness of the $\mathrm{SiO}_{2}$ shell. This shift is ascribed to the modification of the local dielectric environment by the $\mathrm{SiO}_{2}$ shell. ${ }^{35}$ Figure $\mathrm{S} 6$ shows the UV-vis absorption spectra for thin films of Au@SiO $\mathrm{SPs}_{2}$ deposited with the spray pyrolysis method. A small shift of the absorption maximum to the red side is also observed in the films with thinner layer of $\mathrm{SiO}_{2}$. At thicker layer of the $\mathrm{SiO}_{2}$ shell, the absorption spectra are very similar to those in solution. The shift in the absorption signal might be ascribed to a weak plasmon coupling in aggregated Au@SiO 2 NPs in lower values of $\mathrm{SiO}_{2}$ thickness. After the characterization of the $\mathrm{SiO}_{2}$ layer, the $\mathrm{Au} @ \mathrm{SiO}_{2} \mathrm{NPs}$ are deposited on a glass substrate via the spray pyrolysis method. Figure S7 displays the SEM images of layers of Au NPs prepared from three different concentrations of the initial Au NPs solution. In all three SEM images, the Au NPs are homogeneously distributed throughout the substrate but upon increasing the concentration of the Au NPs solution, the distance between the $\mathrm{Au}$ NPs is shorter occupying the wide majority of the area of the substrate. Thus, the separation in the last two samples (most concentrated ones) is on average about $50 \mathrm{~nm}$ which is smaller than the charge carrier diffusion length in the MAPbl 3 perovskite. Figure S7 also shows the elemental analysis of Au for the three SEM images revealing the regular distribution of Au element throughout the substrate.

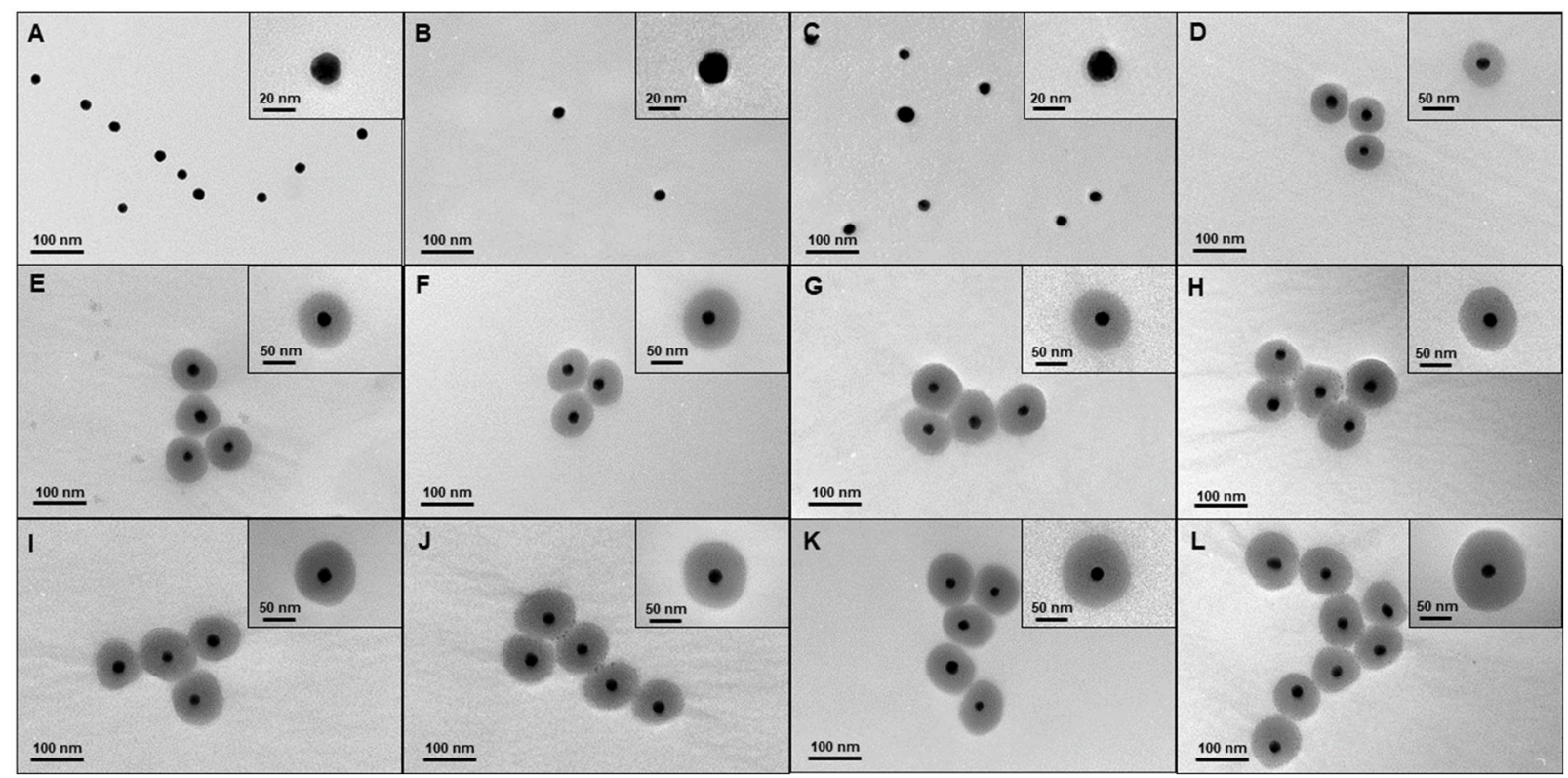


Figure 2. Representative transmission electron microscopy (TEM) images of bare Au NPs (A), and silica-coated Au NPs with shells after 0.25 (B), 0.66 (C), 1 (D), $3(\mathrm{E}), 6(\mathrm{~F}), 12(\mathrm{G}), 24(\mathrm{H}), 48(\mathrm{I}), 72(\mathrm{~J}), 96(\mathrm{~K})$ and $120(\mathrm{~L})$ hours of Stöber growth with resulting average thickness of $3,7,18,23,28,33,37,39,41,43$ and $45 \mathrm{~nm}$, respectively.

Once the distance between the Au NPs and the MAPbl 3 layers is finely controlled through the $\mathrm{SiO}_{2}$ shell, the changes in the $\mathrm{PL}$ intensity of the MAPbl 3 perovskite were studied. Figure 3 displays the $\mathrm{PL}$ spectra of $\mathrm{MAPbl}_{3}$ perovskite films deposited on top of $\mathrm{Au} @ \mathrm{SiO}_{2} \mathrm{NPs}$ layers with different $\mathrm{SiO}_{2}$ thickness. The PL spectrum of the sample prepared using bare Au NPs exhibits an intense quenching with a $98 \%$ reduction of the PL signal with respect to the reference $\mathrm{MAPb}_{3}$ film without plasmonic NPs. It is worth noting that no changes in the shape and position of the PL peak were observed. The high quenching efficiency is a clear evidence of the strong interaction between the plasmonic NPs and the perovskite film which might be attributed to electronic recombination at the surface of the Au NPs or energy transfer to the metal NPs. Moreover, the plasmonic near-field of metal NPs may also impact on the exciton dissociation as, for example, reducing the exciton binding energy. ${ }^{13}$ Remarkably, in the samples with the Au@SiO 2 NPs, the PL signal is gradually recovered when increasing the thickness of the $\mathrm{SiO}_{2}$ shell with a full recovery at $\sim 43 \mathrm{~nm}$ separation between both materials imposed by the $\mathrm{SiO}_{2}$ shell. The long distance at which this non-radiative mechanism still operates, and the clear distance-dependence of the PL quenching is a strong evidence for a nanometal surface energy transfer (NSET) process where, unlike the short range of action of the Förster energy transfer $(1-10 \mathrm{~nm})$, the dipole-surface interactions are active at longer distances (1-30 nm). ${ }^{36}$ Given the value of thickness of the silica layer was tuned to control the distance between the surface of the Au NP and the perovskite material, the effect of the surface plasmon resonance from the Au NP is completely neglected after reaching a given value of thickness, indicating no interaction Au NPperovskite. Thus, no field enhancement after a certain $\mathrm{SiO}_{2}$ thickness was found. Additionally, the notable quenching of the PL signal at moderate distances excludes an electron recombination process as the mechanism responsible for 
the PL quenching. Indeed, the isolating and photoinert layer of $\mathrm{SiO}_{2}$ around the $\mathrm{Au}$ NPs hampers the existence of electronic coupling between both active materials.

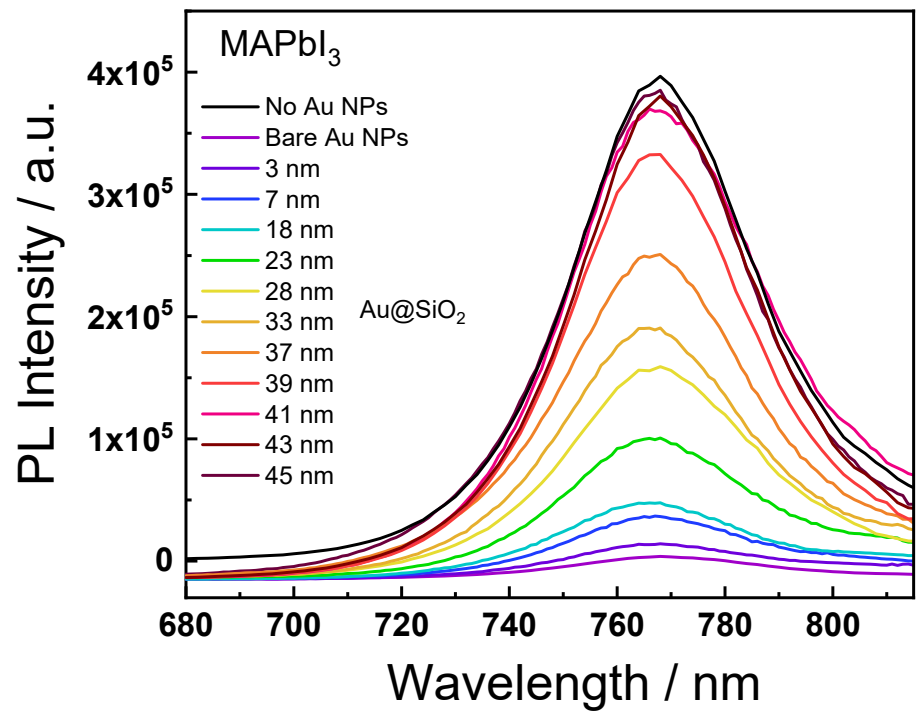

Figure 3. $\mathrm{PL}$ intensity of the MAPbl 3 hybrid perovskite film deposited on top of a layer of $\mathrm{Au} @ \mathrm{SiO}_{2} \mathrm{NPs}$ with different $\mathrm{SiO}_{2}$ thickness. $\lambda$ exc $=400 \mathrm{~nm}$.

We have also measured the time constants $(\tau)$ related to the decay of the PL signal of the $\mathrm{MAPbl}_{3}$ perovskite. Table $\mathrm{S} 1$ includes the average values calculated by using a triexponential function to fit the experimental data. Following the same tendency observed in the steady-state PL spectra, the time constant is significantly shorter in the samples with the bare Au NPs with respect to the reference $\mathrm{MAPbl}_{3}$ hybrid perovskite. Moreover, longer time constants are gradually obtained when increasing the thickness of the $\mathrm{SiO}_{2}$ shell in the $\mathrm{Au} @ \mathrm{SiO}_{2}$ NPs. The radiative and non-radiative rate constants ( $k_{\mathrm{rad}}$ and $\left.k_{\mathrm{nrad}}\right)$ for 
each sample can be calculated with the measured time constants and the PL quantum yield using the following equations:

$$
k_{r a d}=\frac{\Phi_{P L}}{\tau_{P L}} \quad \text { (1) } \quad k_{n r a d}=\frac{1}{\tau_{P L}}-k_{r a d}
$$

Figure 4A displays the obtained values of $k_{\text {rad }}$ with minor changes throughout all samples while $k_{\text {nrad }}$ decreases upon growing the thickness of the $\mathrm{SiO}_{2}$ shell. Thus, the impact of the surface plasmon of the Au NPs on the MAPbl 3 perovskite is demonstrated to occur through a non-radiative mechanism, as the previously mentioned NSET process. ${ }^{36}$

To prove the operation of the NSET mechanism in the non-radiative deactivation of the MAPbl 3 perovskite in the presence of the $\mathrm{Au} @ \mathrm{SiO}_{2} \mathrm{NPs}$, the experimental $\mathrm{PL}$ quenching efficiency is modeled by using a theoretical inverse four power distance dependence between the perovskite excitons and the Au NP surface (equation 3) as stated in the CPS-Kuhn model. ${ }^{37,38}$

$$
E_{E n T}=\frac{1}{1+\left(\frac{d}{d_{0}}\right)^{4}}
$$

Where $d$ is the separation between the MAPbl 3 perovskite and the Au NPs defined by the thickness of the $\mathrm{SiO}_{2}$ shell and $d_{0}$ is the distance at which the probability of non-radiative quenching of the MAPbl 3 perovskite leads to a $50 \%$ lowering of the PL intensity. The parameters defining the value of $d_{0}$ are described by the CPS-Kuhn theory applied to thin films accounting for the size-dependent electronic properties of the Au NPs (equation 4).

$$
d_{0}=\frac{\alpha \lambda}{n_{m}}\left(A \Phi_{P L}\right)^{1 / 4}\left(\frac{n_{r}}{2 n_{m}}\left(1+\frac{\varepsilon_{1}^{2}}{\left|\epsilon_{2}\right|^{2}}\right)\right)^{1 / 4}
$$


The do value incorporates the donor ( $\left.\mathrm{MAPbl}_{3}\right)$ and acceptor (Au NPs) terms that determines the extension of the energy transfer mechanism: $\lambda$ is the emission wavelength maximum of the MAPbl${ }_{3}(775 \mathrm{~nm}), \Phi_{\mathrm{PL}}$ is the $\mathrm{PL}$ quantum yield of the $\mathrm{MAPbl}_{3}(0.03), A$ is the absorptivity of the Au NPs layers, $n_{\mathrm{r}}$ is the refractive index of the metal (0.52), $\varepsilon_{2}$ is the complex dielectric function of the metal, $\varepsilon_{1}$ is the solvent dielectric (air), $\mathrm{n}_{\mathrm{m}}$ is the index of refraction (air) and $\alpha$ is the orientation of the $\mathrm{MAPbl}_{3}$ to the metal plasmon vector $(0.116)$. The $\varepsilon_{2}$ and $A$ values are calculated according to the CPS-Kuhn model to account for their dependence with the size of the Au NPs. ${ }^{36,39}$
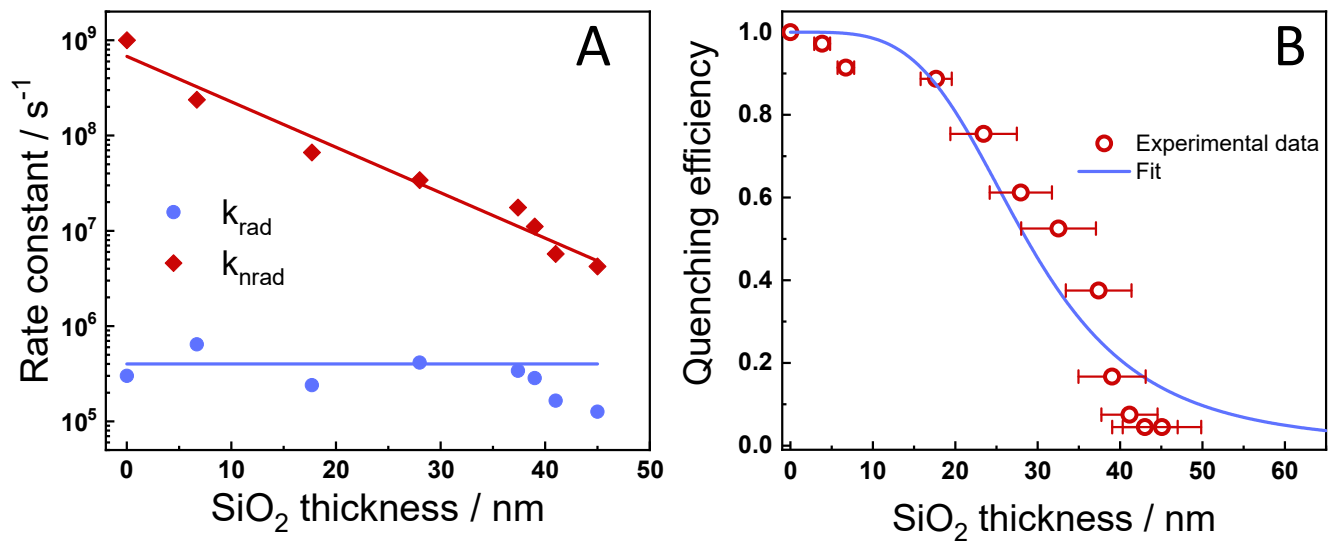

Figure 4. A) Radiative and non-radiative rate constant of the $\mathrm{MAPbl}_{3}$ hybrid perovskite film deposited on top of a layer of $\mathrm{Au} @ \mathrm{SiO}_{2} \mathrm{NPs}$ with different $\mathrm{SiO}_{2}$ thicknesses. The $k_{\mathrm{rad}}$ and $k_{\mathrm{n} r a d}$ are calculated using equation (1) and (2). The solid lines in the figure are only guide for the eye. B) Experimental data of the quenching efficiency obtained from PL spectra in Figure 3 (red squares) and fit of the data using the CPS-Kuhn model for Au NPs with a radius of $10 \mathrm{~nm}$ (blue line).

Figure 4B shows the fit of the experimental values of quenching efficiency at the different thickness of the $\mathrm{SiO}_{2}$ shell using the CPS-Kuhn model. A good agreement of the data with the fit is observed although the model overestimates the quenching at large thickness of the $\mathrm{SiO}_{2}$ shell $(40-45 \mathrm{~nm})$. Thus, our results indicate that the nanometal surface energy transfer is the more suitable mechanism to account for the PL quenching observed in the PeSCs. The surface energy transfer process has been widely investigated as a deactivation 
mechanism in fluorescence molecules/Au NPs with applications in biophysics of molecular chemistry. The validity of the model for the hybrid perovskites extends the range of operation of this mechanism and open the way for further investigations in this field.

\section{CONCLUSIONS}

In summary, by constructing a series of core-shell Au@SiO ${ }_{2} \mathrm{NPs}$ with tunable thickness of the optically inert $\mathrm{SiO}_{2}$ shell, the influence of the plasmonic field on the photophysical properties of $\mathrm{MAPb}_{3}$ perovskite thin films has been carefully characterized. The PL intensity is entirely quenched in the presence of Au NPs but it gradually recovers its initial intensity at increasing thickness of the $\mathrm{SiO}_{2}$ shell. The radiative rate constant is calculated to keep invariable for all samples while the non-radiative rate constant decreases at thicker $\mathrm{SiO}_{2}$ thickness. $\mathrm{A}$ nanometal surface energy transfer process is proved to be responsible for the non-radiative $\mathrm{PL}$ quenching of the $\mathrm{MAPb}_{3}$ hybrid perovskite. The enhanced absorption in PSCs due to light scattering of the Au NPs is a well-established effect that should prevail over the energy transfer reaction to optimize the performance. Our outcomes reveal the importance of the $\mathrm{SiO}_{2}$ shell to prevent the detrimental energy transfer process in PSCs decorated with Au NPs. ${ }^{28}$

\section{Acknowledgements}

S.R.-T. thanks the Ministry of Education, Culture, and Sport for a FPU fellowship (FPU18/04452). This work was financially supported by the Ministerio de Economía y Competitividad (MINECO) of Spain, through CTQ2017-84221-R, CTQ2017-83961-R, and CTQ2017-92264-EXP projects, as well as by the Andalusian Government of Spain, through UCO-1265871-R and UCO-1263193 projects, all cofinanced with FEDER funds. Authors also acknowledge to the SCAI-UCO service (especially Francisco García-Alfonso) for the assistance with TEM and SEM analysis. 


\section{Bibliography}

(1) Stranks, S. D.; Snaith, H. J. Metal-Halide Perovskites for Photovoltaic and Light-Emitting Devices. Nat. Nanotechnol. 2015, 10 (5), 391-402. https://doi.org/10.1038/nnano.2015.90.

(2) Yang, W. S.; Park, B. W.; Jung, E. H.; Jeon, N. J.; Kim, Y. C.; Lee, D. U.; Shin, S. S.; Seo, J.; Kim, E. K.; Noh, J. H.; Seok, S. II. Iodide Management in Formamidinium-Lead-Halide-Based Perovskite Layers for Efficient Solar Cells. Science (80-. ). 2017, 356 (6345), 1376-1379. https://doi.org/10.1126/science.aan2301.

(3) Cao, Y.; Wang, N.; Tian, H.; Guo, J.; Wei, Y.; Chen, H.; Miao, Y.; Zou, W.; Pan, K.; He, Y.; Cao, H.; Ke, Y.; Xu, M.; Wang, Y.; Yang, M.; Du, K.; Fu, Z.; Kong, D.; Dai, D.; Jin, Y.; Li, G.; Li, H.; Peng, Q.; Wang, J.; Huang, W. Perovskite Light-Emitting Diodes Based on Spontaneously Formed Submicrometre-Scale Structures. Nature 2018, 562 (7726), 249-253. https://doi.org/10.1038/s41586-018-0576-2.

(4) Lin, K.; Xing, J.; Quan, L. N.; de Arquer, F. P. G.; Gong, X.; Lu, J.; Xie, L.; Zhao, W.; Zhang, D.; Yan, C.; Li, W.; Liu, X.; Lu, Y.; Kirman, J.; Sargent, E. H.; Xiong, Q.; Wei, Z. Perovskite Light-Emitting Diodes with External Quantum Efficiency Exceeding 20 per Cent. Nature 2018, 562 (7726), 245-248. https://doi.org/10.1038/s41586-018-0575-3.

(5) Wei, H.; Fang, Y.; Mulligan, P.; Chuirazzi, W.; Fang, H. H.; Wang, C.; Ecker, B. R.; Gao, Y.; Loi, M. A.; Cao, L.; Huang, J. Sensitive X-Ray Detectors Made of Methylammonium Lead Tribromide Perovskite Single 
Crystals. Nat. Photonics 2016, 10 (5), 333-339.

https://doi.org/10.1038/nphoton.2016.41.

(6) He, Y.; Matei, L.; Jung, H. J.; McCall, K. M.; Chen, M.; Stoumpos, C. C.; Liu, Z.; Peters, J. A.; Chung, D. Y.; Wessels, B. W.; Wasielewski, M. R.; Dravid, V. P.; Burger, A.; Kanatzidis, M. G. High Spectral Resolution of Gamma-Rays at Room Temperature by Perovskite CsPbBr3 Single Crystals. Nat. Commun. 2018, 9 (1), 1-8. https://doi.org/10.1038/s41467018-04073-3.

(7) Eaton, S. W.; Lai, M.; Gibson, N. A.; Wong, A. B.; Dou, L.; Ma, J.; Wang, L. W.; Leone, S. R.; Yang, P. Lasing in Robust Cesium Lead Halide Perovskite Nanowires. Proc. Natl. Acad. Sci. U. S. A. 2016, 113 (8), 1993-1998. https://doi.org/10.1073/pnas.1600789113.

(8) Zhu, H.; Fu, Y.; Meng, F.; Wu, X.; Gong, Z.; Ding, Q.; Gustafsson, M. V.; Trinh, M. T.; Jin, S.; Zhu, X. Y. Lead Halide Perovskite Nanowire Lasers with Low Lasing Thresholds and High Quality Factors. Nat. Mater. 2015, 14 (6), 636-642. https://doi.org/10.1038/nmat4271.

(9) Li, R.; Li, X.; Wu, J.; Lv, X.; Zheng, Y. Z.; Zhao, Z.; Ding, X.; Tao, X.; Chen, J. F. Few-Layer Black Phosphorus-on-MAPbI3 for Superb VisibleLight Photocatalytic Hydrogen Evolution from HI Splitting. Appl. Catal. B Environ. 2019, 259 (June), 118075. https://doi.org/10.1016/j.apcatb.2019.118075.

(10) Huang, H.; Pradhan, B.; Hofkens, J.; Roeffaers, M. B. J.; Steele, J. A. Solar-Driven Metal Halide Perovskite Photocatalysis: Design, Stability, and Performance. ACS Energy Lett. 2020, 5 (4), 1107-1123. https://doi.org/10.1021/acsenergylett.0c00058.

(11) Lu, H.; Wang, J.; Xiao, C.; Pan, X.; Chen, X.; Brunecky, R.; Berry, J. J.; Zhu, K.; Beard, M. C.; Vardeny, Z. V. Spin-Dependent Charge Transport through 2D Chiral Hybrid Lead-lodide Perovskites. Sci. Adv. 2019, 5 (12), 1-8. https://doi.org/10.1126/sciadv.aay0571.

(12) Wang, T.; Yue, D.; Li, X.; Zhao, Y. Lead-Free Double Perovskite Cs2AgBiBr6/RGO Composite for Efficient Visible Light Photocatalytic $\mathrm{H} 2$ Evolution. Appl. Catal. B Environ. 2020, 268 (September 2019), 118399. https://doi.org/10.1016/j.apcatb.2019.118399.

(13) Zhang, W.; Saliba, M.; Stranks, S. D.; Sun, Y.; Shi, X.; Wiesner, U.; 
Snaith, H. J. Enhancement of Perovskite-Based Solar Cells Employing Core-Shell Metal Nanoparticles. Nano Lett. 2013, 13 (9), 4505-4510. https://doi.org/10.1021/nl4024287.

(14) Li, S.; Miao, P.; Zhang, Y.; Wu, J.; Zhang, B.; Du, Y.; Han, X.; Sun, J.; Xu, P. Recent Advances in Plasmonic Nanostructures for Enhanced Photocatalysis and Electrocatalysis. Adv. Mater. 2020, 2000086, 1-19. https://doi.org/10.1002/adma.202000086.

(15) Mosquera, J.; Zhao, Y.; Jang, H. J.; Xie, N.; Xu, C.; Kotov, N. A.; LizMarzán, L. M. Plasmonic Nanoparticles with Supramolecular Recognition. Adv. Funct. Mater. 2020, 30 (2), 1-17. https://doi.org/10.1002/adfm.201902082.

(16) de Aberasturi, D. J.; Serrano-Montes, A. B.; Liz-Marzán, L. M. Modern Applications of Plasmonic Nanoparticles: From Energy to Health. Adv. Opt. Mater. 2015, 3 (5), 602-617. https://doi.org/10.1002/adom.201500053.

(17) Espinosa, N.; Serrano-Luján, L.; Urbina, A.; Krebs, F. C. Solution and Vapour Deposited Lead Perovskite Solar Cells: Ecotoxicity from a Life Cycle Assessment Perspective. Sol. Energy Mater. Sol. Cells 2015, 137, 303-310. https://doi.org/10.1016/j.solmat.2015.02.013.

(18) Yao, K.; Zhong, H.; Liu, Z.; Xiong, M.; Leng, S.; Zhang, J.; Xu, Y. X.; Wang, W.; Zhou, L.; Huang, H.; Jen, A. K. Y. Plasmonic Metal Nanoparticles with Core-Bishell Structure for High-Performance Organic and Perovskite Solar Cells. ACS Nano 2019, 13 (5), 5397-5409. https://doi.org/10.1021/acsnano.9b00135.

(19) Saliba, M.; Zhang, W.; Burlakov, V. M.; Stranks, S. D.; Sun, Y.; Ball, J. M.; Johnston, M. B.; Goriely, A.; Wiesner, U.; Snaith, H. J. Plasmonic-Induced Photon Recycling in Metal Halide Perovskite Solar Cells. Adv. Funct. Mater. 2015, 25 (31), 5038-5046. https://doi.org/10.1002/adfm.201500669.

(20) Wu, R.; Yang, B.; Zhang, C.; Huang, Y.; Cui, Y.; Liu, P.; Zhou, C.; Hao, Y.; Gao, Y.; Yang, J. Prominent Efficiency Enhancement in Perovskite Solar Cells Employing Silica-Coated Gold Nanorods. J. Phys. Chem. C 2016, 120 (13), 6996-7004. https://doi.org/10.1021/acs.jpcc.6b00309.

(21) Carretero-Palacios, S.; Jiménez-Solano, A.; Míguez, H. Plasmonic 
Nanoparticles as Light-Harvesting Enhancers in Perovskite Solar Cells: A User's Guide. ACS Energy Lett. 2016, 1 (1), 323-331.

https://doi.org/10.1021/acsenergylett.6b00138.

(22) Cui, X.; Cui, X.; Chen, Y.; Zhang, M.; Harn, Y. W.; Qi, J.; Gao, L.; Wang, Z. L.; Huang, J.; Yang, Y.; Lin, Z. Tailoring Carrier Dynamics in Perovskite Solar Cells: Via Precise Dimension and Architecture Control and Interfacial Positioning of Plasmonic Nanoparticles. Energy Environ. Sci. 2020, 13 (6), 1743-1752. https://doi.org/10.1039/c9ee03937f.

(23) Li, Y. F.; Kou, Z. L.; Feng, J.; Sun, H. B. Plasmon-Enhanced Organic and Perovskite Solar Cells with Metal Nanoparticles. Nanophotonics 2020, 9 (10), 3111-3133. https://doi.org/10.1515/nanoph-2020-0099.

(24) Carretero-Palacios, S.; Calvo, M. E.; Míguez, H. Absorption Enhancement in Organic-Inorganic Halide Perovskite Films with Embedded Plasmonic Gold Nanoparticles. J. Phys. Chem. C 2015, 119 (32), 18635-18640. https://doi.org/10.1021/acs.jpcc.5b06473.

(25) Jacak, W. A.; Jacak, J. E. New Channel of Plasmon Photovoltaic Effect in Metalized Perovskite Solar Cells. J. Phys. Chem. C 2019, 123 (50), 30633-30639. https://doi.org/10.1021/acs.jpcc.9b09763.

(26) Lu, Z.; Pan, X.; Ma, Y.; Li, Y.; Zheng, L.; Zhang, D.; Xu, Q.; Chen, Z.; Wang, S.; Qu, B.; Liu, F.; Huang, Y.; Xiao, L.; Gong, Q. PlasmonicEnhanced Perovskite Solar Cells Using Alloy Popcorn Nanoparticles. RSC Adv. 2015, 5 (15), 11175-11179.

https://doi.org/10.1039/c4ra16385k.

(27) Wu, B.; Wu, X.; Guan, C.; Tai, K. F.; Yeow, E. K. L.; Fan, H. J.; Mathews, N.; Sum, T. C. Uncovering Loss Mechanisms in Silver NanoparticleBlended Plasmonic Organic Solar Cells. Nat. Commun. 2013, 4 (May), 17. https://doi.org/10.1038/ncomms3004.

(28) Alcocer, M. J. P.; Leijtens, T.; Herz, L. M.; Petrozza, A.; Snaith, H. J. Electron-Hole Diffusion Lengths Exceeding Trihalide Perovskite Absorber. Science (80-. ). 2013, 342 (October), 341-344.

https://doi.org/10.1126/science.1243982.

(29) Deng, S.; Zhang, B.; Choo, P.; Smeets, P. J. M.; Odom, T. W. Plasmonic Photoelectrocatalysis in Copper-Platinum Core-Shell Nanoparticle Lattices. Nano Lett. 2021, 21 (3), 1523-1529. 
https://doi.org/10.1021/acs.nanolett.0c05029.

(30) Giner-Casares, J. J.; Henriksen-Lacey, M.; García, I.; Liz-Marzán, L. M. Plasmonic Surfaces for Cell Growth and Retrieval Triggered by NearInfrared Light. Angew. Chemie - Int. Ed. 2016, 55 (3), 974-978. https://doi.org/10.1002/anie.201509025.

(31) Zhang, X.; Marocico, C. A.; Lunz, M.; Gerard, V. A.; Gun'Ko, Y. K.; Lesnyak, V.; Gaponik, N.; Susha, A. S.; Rogach, A. L.; Bradley, A. L. Wavelength, Concentration, and Distance Dependence of Nonradiative Energy Transfer to a Plane of Gold Nanoparticles. ACS Nano 2012, 6 (10), 9283-9290. https://doi.org/10.1021/nn303756a.

(32) Pang, G. A.; Poisson, F.; Laufer, J.; Haisch, C.; Bossy, E. Theoretical and Experimental Study of Photoacoustic Excitation of Silica-Coated Gold Nanospheres in Water. J. Phys. Chem. C 2020. https://doi.org/10.1021/acs.jpcc.9b09040.

(33) Vanderkooy, A.; Chen, Y.; Gonzaga, F.; Brook, M. A. Silica Shell/Gold Core Nanoparticles: Correlating Shell Thickness with the Plasmonic Red Shift upon Aggregation. ACS Appl. Mater. Interfaces 2011, 3 (10), 39423947. https://doi.org/10.1021/am200825f.

(34) Liz-marza, L. M.; Giersig, M.; Mulvaney, P. Synthesis of Nanosized Gold Silica Core - Shell Particles. 1996, 7463 (5), 4329-4335.

(35) Gao, C.; Lyu, F.; Yin, Y. Encapsulated Metal Nanoparticles for Catalysis. Chem. Rev. 2020. https://doi.org/10.1021/acs.chemrev.0c00237.

(36) Breshike, C. J.; Riskowski, R. A.; Strouse, G. F. Leaving Förster Resonance Energy Transfer behind: Nanometal Surface Energy Transfer Predicts the Size-Enhanced Energy Coupling between a Metal Nanoparticle and an Emitting Dipole. J. Phys. Chem. C 2013, 117 (45), 23942-23949. https://doi.org/10.1021/jp407259r.

(37) Kuhn, H. Classical Aspects of Energy Transfer in Molecular Systems. J. Chem. Phys. 1970, 53 (1), 101-108. https://doi.org/10.1063/1.1673749.

(38) Miller, D. T. Comments on the Classical Theory of Magnetic Monopoles. Math. Proc. Cambridge Philos. Soc. 1971, 69 (3), 449-456. https://doi.org/10.1017/S0305004100046892.

(39) Jain, P. K.; Lee, K. S.; El-Sayed, I. H.; El-Sayed, M. A. Calculated Absorption and Scattering Properties of Gold Nanoparticles of Different 
Size, Shape, and Composition: Applications in Biological Imaging and Biomedicine. J. Phys. Chem. B 2006, 110 (14), 7238-7248. https://doi.org/10.1021/jp057170o. 